\title{
Nanoscale
}

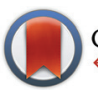

CrossMark $\leftarrow$ click for updates

Cite this: Nanoscale, 2015, 7, 20009

Received 26th August 2015,

Accepted 29th October 2015

DOI: 10.1039/c5nr05820a

www.rsc.org/nanoscale

\section{All-solution processed transparent organic light emitting diodes}

\author{
Min Zhang, $\uparrow$ Stefan Höfle, ${ }^{\star} \dagger$ Jens Czolk, Adrian Mertens and Alexander Colsmann*
}

In this work, we report on indium tin oxide-free, all-solution processed transparent organic light emitting diodes (OLEDs) with inverted device architecture. Conductive polymer layers are employed as both transparent cathodes and transparent anodes, with the top anodes having enhanced conductivities from a supporting stochastic silver nanowire mesh. Both electrodes exhibit transmittances of $80-90 \%$ in the visible spectral regime. Upon the incorporation of either yellow- or blue-light emitting fluorescent polymers, the OLEDs show low onset voltages, demonstrating excellent charge carrier injection from the polymer electrodes into the emission layers. Overall luminances and current efficiencies equal the performance of opaque reference OLEDs with indium tin oxide and aluminium electrodes, proving excellent charge carrier-to-light conversion within the device.

\section{Introduction}

Today, vacuum processed organic light emitting diodes (OLEDs) are often employed for mobile phones or status displays, featuring high contrast and low power consumption. With respect to future lighting applications and the reduction of fabrication costs, large-area printing is widely considered an interesting alternative fabrication method. Whereas solution deposition of multi-layer architectures has been thoroughly investigated for organic solar cells, wet-processed advanced OLED architectures comprising several functional layers with thicknesses of a few $10 \mathrm{~nm}$ have been studied only recently, e.g., in tandem light-emitting devices. ${ }^{1-4}$ The main challenges for the fabrication of multi-layer OLEDs from solution are (i) the sequential deposition of homogenous submicron thinfilms, (ii) to ensure the integrity of the previously applied layers and (iii) to avoid their dissolution upon subsequent deposition of further functional layers. Across all platforms, the solution deposition of the electrodes is an important enabler towards all-printed optoelectronic devices. ${ }^{5-7}$ Besides the high costs for indium, high process temperatures and its brittleness render the commonly used transparent indium tin oxide (ITO) electrodes unsuitable for future roll-to-roll production. To replace the transparent ITO electrode, carbon nanotubes, conductive polymers and metal gratings were widely investigated. ${ }^{8-11}$ In particular, the conductive and mechanical flexible polymer poly(3,4-ethylenedioxy-thiophene):

Light Technology Institute, Karlsruhe Institute of Technology (KIT), Engesserstrasse 13, 76131 Karlsruhe, Germany. E-mail: stefan.hoefle@kit.edu,

alexander.colsmann@kit.edu

$\dagger$ The first two authors contributed equally to this work. poly(styrene sulfonate) (PEDOT:PSS) has been investigated in organic devices either as anode or as cathode. ${ }^{12-16}$ By adding high boiling point solvents such as glycerol, dimethyl sulfoxide (DMSO) or ethylene glycol to the PEDOT:PSS solution, the bottom electrode conductivity can be further enhanced. ${ }^{10,17,18}$ Transparent optoelectronic devices have been developed by replacing opaque metal top electrodes by PEDOT:PSS. ${ }^{14,15,19-22}$ Replacing both electrodes with laterally structured conductive polymer layers in OLEDs, however, remains challenging due to the physical interaction of both water soluble electrodes and the influence of the PEDOT:PSS on the electron-to-photon conversion in the emission layer.

In this work, we investigate all-solution processed, fluorescent transparent OLEDs (TOLEDs). Preserving the excellent device efficiency, we replace both the top and the bottom electrodes by the conductive polymer PEDOT:PSS, thereby overcoming the subtle difficulty of depositing the PEDOT:PSS top electrode atop a layer stack that comprises a water soluble bottom electrode. The conductivity of the PEDOT:PSS top electrode was enhanced by a stochastic silver nanowire (AgNW) mesh. A bilayer from polyethylenimine (PEI) and zinc oxide ( $\mathrm{ZnO})$ enables efficient electron injection from the bottom PEDOT:PSS cathode into the emission layer, whereas a tungsten oxide $\left(\mathrm{WO}_{3}\right)$ layer facilitates hole injection from the top PEDOT:PSS anode. ${ }^{21,23-25}$

\section{Results and discussion}

TOLEDs were fabricated according to the inverted PEDOT:PSS/ $\mathrm{ZnO} / \mathrm{PEI} / \mathrm{Super}$ Yellow/WO $/ \mathrm{WO}_{3} / \mathrm{PEDOT}$ :PSS/AgNW device architecture illustrated in Fig. 1a. The most important steps of the 
(a)

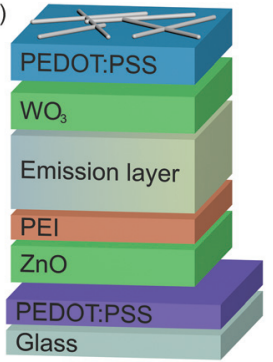

(b)

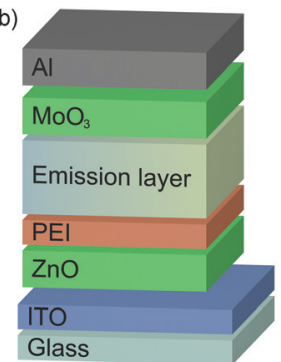

Fig. 1 Device architectures of (a) the all-solution processed TOLEDs comprising PEDOT:PSS bottom cathodes and PEDOT:PSS/AgNW top anodes and (b) the opaque reference OLEDs.

TOLED fabrication procedure are depicted in Fig. 2. Lateral structuring of the conductive PEDOT:PSS layers is key to their employment as electrodes. For the bottom cathode we placed dicing tape atop the spin coated PEDOT:PSS layer and washed away the unprotected parts of the polymer layer. Then the dicing tape was removed. After depositing ZnO, PEI, Super Yellow and $\mathrm{WO}_{3}$ by spin coating, commercially available, prestructured adhesive tape was applied atop the layer stack, with voids defining the shape of the top anode. PEDOT:PSS was then spin coated into the voids of the adhesive tape, not wetting the tape itself. Likewise, an AgNW mesh was drop cast atop the PEDOT:PSS. The PEDOT:PSS anodes were laterally isolated by the adhesive tape, that remains in place, effectively suppressing any cross-talk between the four devices per sample. The cross-sections of the bottom and top PEDOT:PSS electrodes defined the device active areas of $5 \times 5 \mathrm{~mm}^{2}$. A more detailed description of the processing parameters can be found in the Methods section. For reference, we fabricated opaque OLEDs according to the device architecture depicted in Fig. 1b, employing an ITO bottom cathode and a vacuum deposited aluminium top anode. In order to qualify as a replacement for ITO, the PEDOT:PSS bottom electrode has to (a)

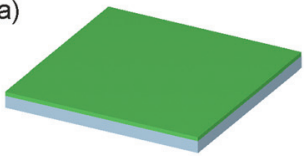

(e)

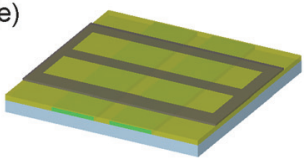

(b)

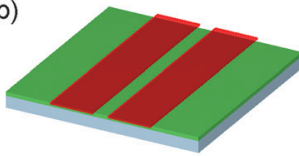

(f)

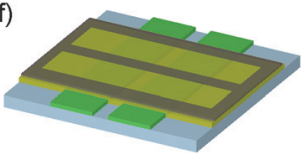

(c)

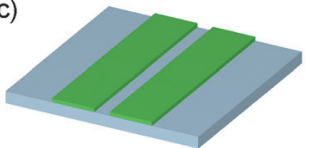

(g)

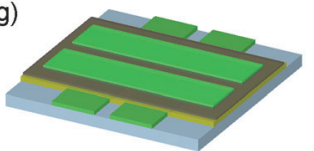

(d)

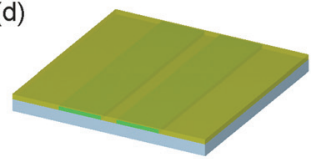

(h)

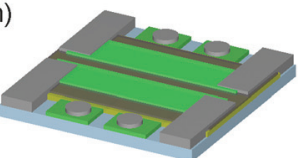

Fig. 2 Illustration of the all-solution TOLED fabrication process: (a) deposition of the conductive PEDOT:PSS electrode, (b) masking of the cathodes by adhesive tape, (c) dissolution of the unprotected PEDOT:PSS and subsequent removal of the tape, (d) deposition of all functional layers other than the electrodes, (e) application of another adhesive tape to define the shape of the anode, (f) outer removal of functional layers to access the bottom cathode, $(\mathrm{g})$ application of the PEDOT:PSS/AgNW top electrode utilizing selective wetting within the adhesive tape voids and (h) application of metal contact pads that do not affect the working principle of the all-solution processed TOLED.
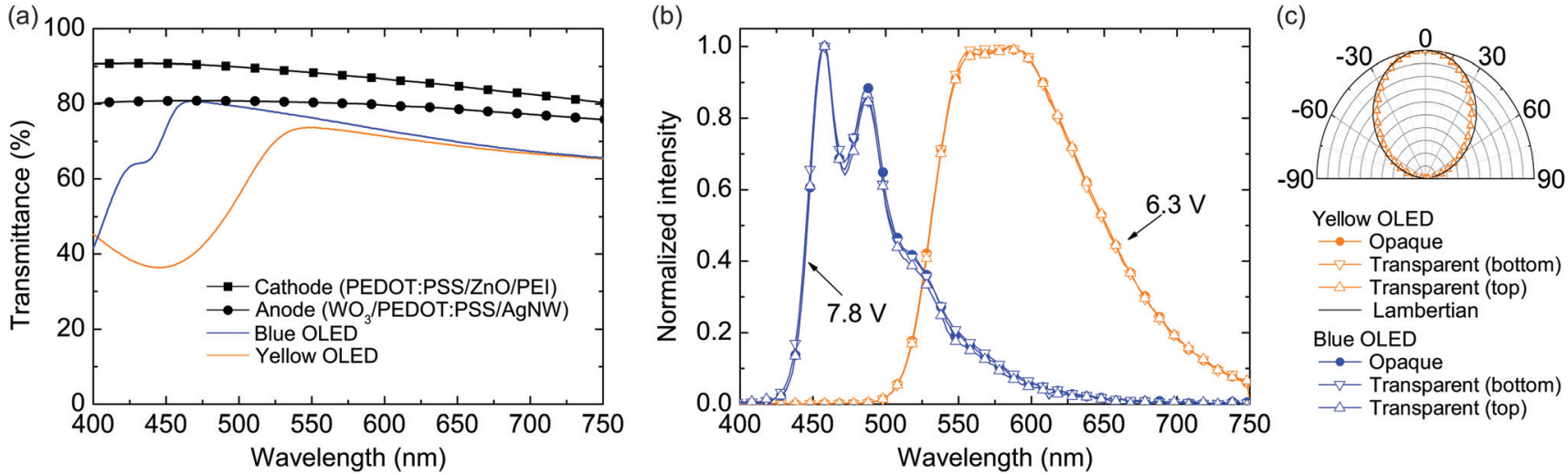

Fig. 3 (a) Typical transmittance spectra of the top and bottom electrodes, and of an entire device comprising either of the light-emitting polymers Super Yellow or Merck Blue. (b) Emission spectra of the yellow and blue reference OLEDs and TOLEDs at $6.3 \mathrm{~V}$ or $7.8 \mathrm{~V}$ driving voltage, respectively. The shapes of the emission spectra in top and bottom direction match the shape of the emission spectra of the respective opaque devices. (c) Lambertian distribution of the light emitted through the PEDOT:PSS/AgNW electrode (Yellow OLED). 
exhibit a reasonable conductivity and transparency. After adding 5 vol\% of DMSO to the PEDOT:PSS solution, the $130 \mathrm{~nm}$ thick PEDOT:PSS layers yielded conductivities of 625 $\mathrm{S} \mathrm{cm}^{-1}$, as determined by four-point probing, which is a common conductivity when employing this process route. ${ }^{14}$ This conductivity corresponds to a sheet resistance of about $123 \Omega \square^{-1}$. To enable electron injection from the high-work function PEDOT:PSS cathode $\left(\Phi_{\mathrm{a}}=5.0 \mathrm{eV}\right.$, as determined by photo-electron yield spectroscopy in air) into the lowest unoccupied molecular orbital (LUMO) of the emitter, an electron injection layer from $\mathrm{ZnO}\left(\Phi_{\mathrm{a}}=4.1 \mathrm{eV}\right.$, as determined by X-ray photo-electron spectroscopy ${ }^{21}$ ) was implemented. Due to the still substantial energetic difference between the work function of $\mathrm{ZnO}$ and the LUMO energy $\left(E_{\mathrm{LuMO}}=-3.0 \mathrm{eV}\right)$ of the emitter polymer Super Yellow, a thin layer of the interface modifier PEI was inserted, yielding a quasi-ohmic contact. ${ }^{21,24}$ The conductivity of the top anode layer was enhanced by a silver nanowire mesh, together exhibiting a very low sheet resistance of 8.5 $\Omega \square^{-1}$. To enhance the injection of holes from the top PEDOT:PSS/AgNW electrode into the emitter polymer, we have deliberately chosen a layer of $\mathrm{WO}_{3}$ which can be applied from a $\mathrm{W}(\mathrm{OEt})_{6}$ precursor, with the conversion process taking place in inert atmosphere at room temperature, thereby protecting the previously applied emission layer from ambient oxygen contamination. The transmissions of both electrodes and the respective adjacent injection layers, i.e., PEDOT:PSS/ZnO/PEI and $\mathrm{WO}_{3} / \mathrm{PEDOT}: \mathrm{PSS} / \mathrm{AgNW}$, were on the order of $80 \%-90 \%$ over the entire visible spectrum as depicted in Fig. 3a. When incorporating a Super Yellow emission layer, the transparency perception of the TOLED was dominated by the emitter polymer, giving the TOLED a distinct yellow colour that is visible in the device photo in Fig. 4. The equal spectral shape of the top and bottom emission of the Super Yellow TOLED at a driving voltage of $6.3 \mathrm{~V}$ is depicted in Fig. 3b, indicating comparable light-outcoupling from the cavity in both directions. The emission in both directions is Lambertian as exemplified

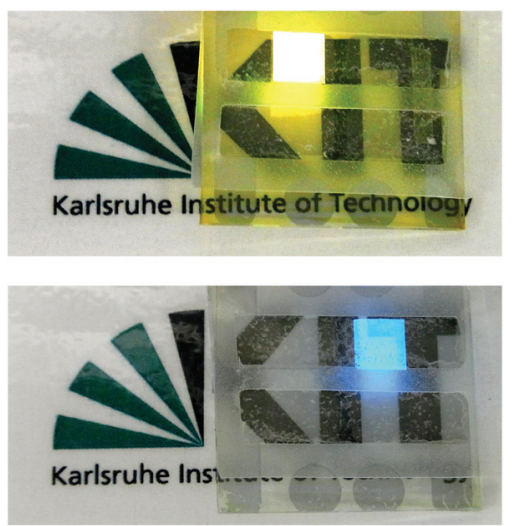

Fig. 4 Photos of the yellow- and the blue-light emitting TOLEDs. The white frames around the emissive areas stem from adhesive tape which was used to structure the top electrode, and then remained within the device. in Fig. 3c. In order to demonstrate the universality of this device concept and to achieve a more colour neutral transparency perception of the OLED, we alternatively incorporated a proprietary blue-emitting, wide-bandgap polymer, hence-
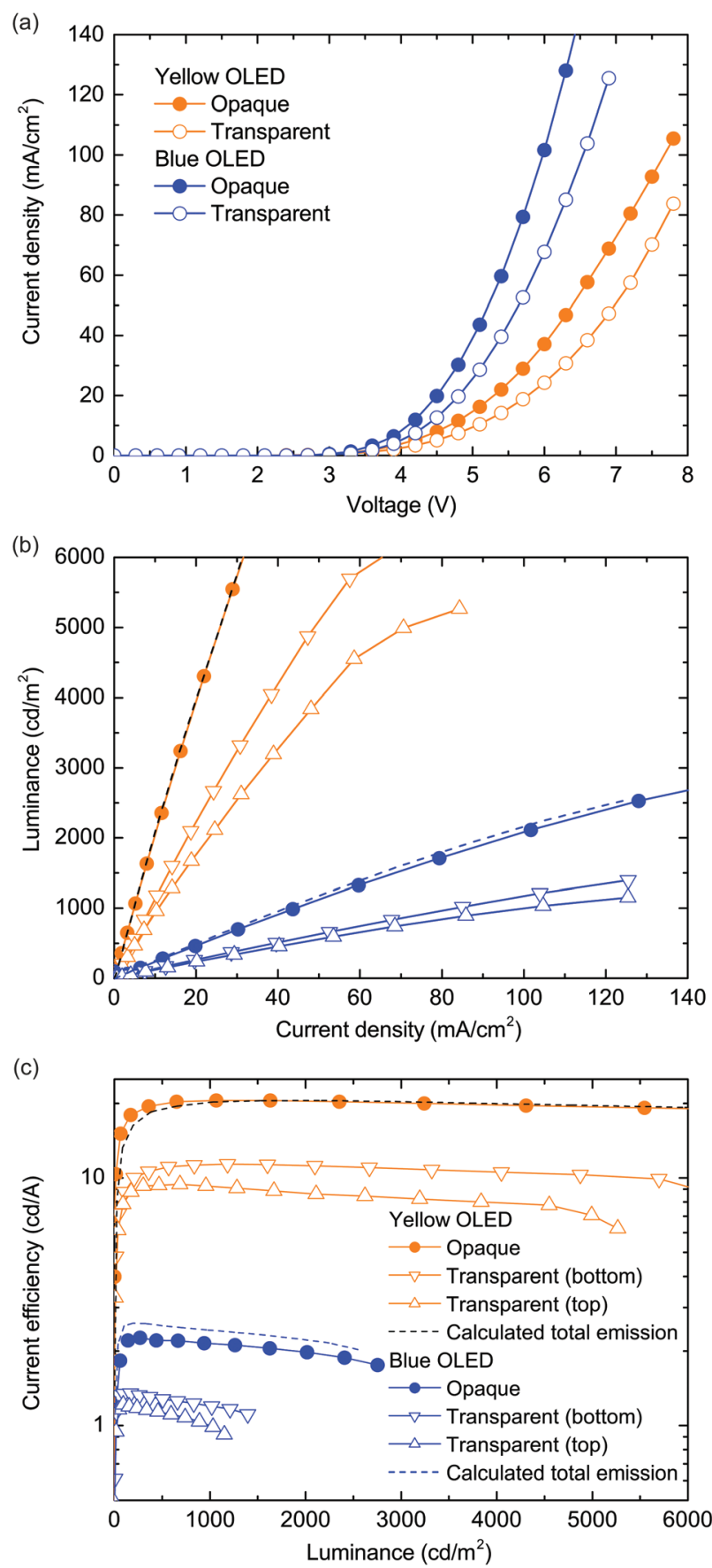

Fig. 5 Optoelectronic characteristics of the yellow- and blue-emitting TOLEDs in comparison to the respective opaque reference devices. In bottom configuration, the light emitted through the glass substrate was collected by the spectrometer, whereas, in top configuration, the light emitted through the anode was detected. (a) Current density vs. voltage $(J-V)$, (b) luminance vs. current density $(L-J)$, (c) current efficiency vs. luminance. 
Table 1 Luminances and current efficiencies of the transparent OLEDs, measured in top and bottom direction at a driving current density of $20 \mathrm{~mA} \mathrm{~cm}{ }^{-2}$. The total emission, as calculated from the top and bottom emission (luminance and current efficiency), matches the emission of the opaque reference devices

\begin{tabular}{lllll}
\hline Material & & Bottom & Top & Total (calculated) \\
\hline Super Yellow & Luminance & $2260 \mathrm{~cd} \mathrm{~m}^{-2}$ & $1760 \mathrm{~cd} \mathrm{~m}^{-2}$ & $4020 \mathrm{~cd} \mathrm{~m}^{-2}$ \\
Merck Blue & Current efficiency & $11.3 \mathrm{~cd} \mathrm{~A}^{-1}$ & $8.8 \mathrm{~cd} \mathrm{~A}^{-1}$ & $20.1 \mathrm{~cd} \mathrm{~A}^{-1}$ \\
& Luminance & $270 \mathrm{~cd} \mathrm{~m}^{-2}$ & $230 \mathrm{~cd} \mathrm{~m}^{-2}$ & $500 \mathrm{~cd} \mathrm{~m}^{-2}$ \\
& Current efficiency & $1.4 \mathrm{~cd} \mathrm{~A}^{-1}$ & $1.1 \mathrm{~cd} \mathrm{~A}^{-1}$ & $2.5 \mathrm{~cd} \mathrm{~A}^{-1}$
\end{tabular}

forth referred to as Merck Blue. The device transparency of $60 \%-80 \%$ over the entire visible spectrum renders the bluelight emitting TOLED suitable for window integration or other applications where colour neutrality and high transparency are major concerns (Fig. 3a).

Fig. 5 depicts the optoelectronic properties of the Super Yellow and Merck Blue TOLEDs (open symbols) and the respective opaque reference devices (closed symbols). Remarkably, the transparent Super Yellow devices exhibit a very low onset voltage of $V_{\mathrm{on}}=2.6 \mathrm{~V}$ (measured at $1 \mathrm{~cd} \mathrm{~m}^{-2}$ ) being only slightly higher than the $V_{\text {on }}=2.3 \mathrm{~V}$ of the opaque reference, hinting at a very good charge carrier injection into the TOLED from both polymer electrodes. Likewise, only a small $V_{\text {on }}$ difference between the reference OLED $\left(V_{\text {on }}=3.1 \mathrm{~V}\right)$ and the transparent device $\left(V_{\mathrm{on}}=3.3 \mathrm{~V}\right)$ was observed when incorporating the blue-light emitting polymer. Towards higher device currents, this difference in driving voltage increases as visible in the $J-V$ curves in Fig. 5a. We attribute the driving voltage increase mainly to the higher series resistance of the bottom polymer cathode. In Fig. 5b, all OLED luminances are shown versus the device current density. Minor differences in the luminances in top (open up-triangles) and bottom (open down-triangles) direction can be attributed to the somewhat different transmission of the top and bottom electrodes. Most importantly, the calculated overall emission (dashed lines), i.e., the combined emission in top and bottom direction, matches the luminance of the opaque reference (closed symbols), proving no charge carrier-to-photon conversion (current efficiency) losses in the all-solution processed devices. The same observation was made for the current efficiency in Fig. 5c. The current efficiency is an overall external quantum efficiency weighted by the spectral sensitivity of the human eye, thereby disregarding resistive losses in the electrodes or injection barriers. The combined top and bottom emission current efficiency of the Super Yellow TOLEDs at a given current density add to $20 \mathrm{~cd} \mathrm{~A}^{-1}$ current efficiency of the opaque reference devices (Table 1). TOLEDs comprising a Merck Blue emission layer qualitatively exhibited the same behaviour, with a lower luminance and current efficiency due to the reduced spectral sensitivity of the human eye in the blue spectral regime. Remarkably, all devices did not show significant roll-offs of the current efficiency, indicating an excellent and balanced charge carrier injection despite the use of two high-work function PEDOT:PSS electrodes.

\section{Conclusions}

We reported on all-solution processed, highly transparent OLEDs with inverted device architectures, comprising PEDOT: PSS top and bottom electrodes, with the conductivity of the top electrode being enhanced by a stochastic silver nanowire mesh. Either yellow- or wide-bandgap, blue-light emitting polymers were used to build the emission layer. The wide-bandgap emitters may be a future gateway to TOLEDs with arbitrary colours through doping with suitable light-emitting dyes. The combined emission in both directions matched the emission of the opaque reference devices, indicating efficient internal charge carrier-to-photon conversion processes. Minor losses in driving voltage originated from the series resistances of the polymer electrodes, which can be compensated by additional bus bars in future device concepts. With respect to the feasibility of the fabrication process and its applicability to future roll-to-roll printing, this device architecture opens up new avenues for all-printed transparent organic light emitting diodes.

\section{Methods}

\section{Device fabrication}

TOLEDs were fabricated according to the device architecture depicted in Fig. 1a, featuring PEDOT:PSS bottom and top electrodes. The all-solution fabrication process is shown in Fig. 2 step-by-step. Prior to the deposition of the polymer bottom cathode, all substrates were successively cleaned with acetone and isopropanol in an ultrasonic bath for $15 \mathrm{~min}$ and subsequently exposed to an oxygen plasma for $2 \mathrm{~min}$ in order to remove organic residues and to polarize the surface for better PEDOT:PSS adhesion. PEDOT:PSS (Clevios PH1000, Heraeus Deutschland $\mathrm{GmbH} \& \mathrm{Co} . \mathrm{KG})$ was filtered with a $0.45 \mu \mathrm{m}$ polyvinylidene fluoride membrane. 5 vol\% DMSO was added to the PEDOT:PSS solution to enhance the layer conductivity, and $10 \mathrm{vol} \%$ isopropanol to improve wetting. After deposition of the PEDOT:PSS layer $(1600 \mathrm{rpm}, 80 \mathrm{~s}$, layer thickness $d=$ $130 \mathrm{~nm}$ ), two stripes of semiconductor wafer processing tape (SWT 20+, Nitto Denko) were applied atop the PEDOT:PSS layer. Then the unprotected PEDOT:PSS was washed away with a moist swab. The wafer tapes were removed, leaving behind structured cathodes. The PEDOT:PSS layer was thermally 
annealed in a vacuum oven at $100{ }^{\circ} \mathrm{C}$ for $15 \mathrm{~min}$. In order to facilitate electron injection, a ZnO and PEI bilayer was applied atop the polymer electrode following processes described in the literature. ${ }^{21} \mathrm{ZnO}$ nanoparticles (Nanograde $\mathrm{N}-10$ ) were spin cast (4000 rpm, $30 \mathrm{~s}, d=30 \mathrm{~nm}$ ) from isopropanol dispersion (0.55 wt\%) under nitrogen atmosphere, followed by thermal annealing (150 $\left.{ }^{\circ} \mathrm{C}, 10 \mathrm{~min}\right)$. The PEI layer was spin coated (5000 rpm, $30 \mathrm{~s}, d=4 \mathrm{~nm}$ ) from 2-methoxyethanol solution (0.4 wt $\%)$ under nitrogen atmosphere. The substrates were annealed in ambient atmosphere $\left(100^{\circ} \mathrm{C}, 10 \mathrm{~min}\right)$ and rinsed with ethanol $(30 \mathrm{~s})$ to remove the PEI surplus. The emitter polymer Super Yellow (Merck KGaA, $E_{\mathrm{LUMO}}=-3.0 \mathrm{eV}, E_{\mathrm{HOMO}}=$ $-5.4 \mathrm{eV}$ ) was spin cast $(1000 \mathrm{rpm}, 45 \mathrm{~s}, d=75 \mathrm{~nm})$ from toluene solution $\left(4 \mathrm{~g} \mathrm{~L}^{-1}\right) .{ }^{26}$ Alternatively, the proprietary blue emitting polymer "Merck Blue" (Merck KGaA, $E_{\text {LUMO }}=-2.9 \mathrm{eV}$, $E_{\text {Hомо }}=-5.5 \mathrm{eV}$ ) was spin cast from anisole $: o$-xylene solution ( $1: 1$ by volume, $8 \mathrm{~g} \mathrm{~L}^{-1}, 1000 \mathrm{rpm}, 60 \mathrm{~s}, d=55 \mathrm{~nm}$ ). The $\mathrm{WO}_{3}$ hole injection layer was deposited from a tungsten(vI) ethoxide precursor solution (W(OEt) 6 , ABCR Dr Braunagel GmbH \& Co. $\mathrm{KG})$ that was diluted in isopropanol $1: 80(\mathrm{v} / \mathrm{v})$, spin cast $(4000$ $\mathrm{rpm}, 30 \mathrm{~s}, d=16 \mathrm{~nm}$ ) and converted in inert atmosphere $(10 \mathrm{~min}) .^{25}$ The counter anode comprised a PEDOT:PSS (Clevios PH1000) layer and a random silver nanowire mesh. Therefore, 5 vol\% of DMSO and 8 vol\% of wetting agents (20 $\mathrm{g} \mathrm{L}^{-1}$ Byk333, Byk Additives \& Instruments, and $20 \mathrm{~g} \mathrm{~L}^{-1}$ Dynol604, Air Products, in isopropanol) were added to the PEDOT:PSS solution to improve wetting. Prior to the deposition of the PEDOT:PSS/AgNW counter electrode, we applied a pre-structured adhesive tape to the device, which later defines the shape of the top anode. The formulation was then spin coated into the voids of the adhesive tape $(1600 \mathrm{rpm}, 80 \mathrm{~s}, d=$ $100 \mathrm{~nm}$ ), followed by an annealing step (100 $\left.{ }^{\circ} \mathrm{C}, 15 \mathrm{~min}\right)$. Subsequently, the silver nanowire dispersion in isopropanol (AgNW, $1.25 \mathrm{~g} \mathrm{~L}^{-1}$, AgNW-115, Seashell Technology) was drop cast on top, yielding an AgNW area density of $0.15 \mathrm{~g} \mathrm{~m}^{-2}$. The devices were annealed $\left(150{ }^{\circ} \mathrm{C}, 5 \mathrm{~min}\right)$ to improve the contact between the silver nanowires and the underlying layer. ${ }^{20}$ For reference, OLEDs with a transparent ITO bottom electrode and an opaque $\mathrm{MoO}_{3} / \mathrm{Al}$ top anode were investigated (Fig. 1b). The indium tin oxide (ITO) coated glass substrates $\left(R_{\square} \approx 13 \Omega \square^{-1}\right)$ were structured in hydrochloric acid, before the other functional layers of the device were applied as described above. $10 \mathrm{~nm}$ of $\mathrm{MoO}_{3}$ and $200 \mathrm{~nm}$ of aluminium were thermally evaporated in high vacuum $\left(10^{-6} \mathrm{mbar}\right)$ to form the counter anode of the reference OLED. ${ }^{27}$ In both OLED architectures, the active area is defined by the intersection of the top and the bottom electrodes $\left(5 \times 5 \mathrm{~mm}^{2}\right)$.

\section{Optoelectronic characterization}

The OLED current density-voltage $(J-V)$ characteristics were recorded with a source measure unit (Keithley 238) at room temperature under inert condition. The emission spectrum was recorded on a Stellar Net EP2000 spectrometer that had been calibrated with a secondary standard calibration halogen lamp (Philips FEL-1000 W). Luminances (cd m${ }^{-2}$ ) were calculated from the emission spectrum assuming Lambertian light distribution. Current efficiencies $\left(\mathrm{cd} \mathrm{A}^{-1}\right)$ were calculated from the electrical and optical properties. Transmittance spectra were measured in a Cary 5000 UV-Vis-NIR spectrophotometer equipped with an integrating sphere (Agilent Technologies). The electrode conductivities were determined by four-point probing under ambient conditions which may lead to an underestimation of the conductivities due to PEDOT:PSS swelling in air. The PEDOT:PSS work function as well as the Super Yellow and Merck Blue HOMO energies were determined by photo-electron yield spectroscopy in air (PESA) on a Rieken Keiki AC-2E. LUMO energies were calculated from the HOMO energies and the optical bandgaps.

\section{Acknowledgements}

This work was supported by the Federal Ministry of Education and Research under contracts no. 13N12281 (project PrintOLED). M.Z. acknowledges funding by the Carl Zeiss Stiftung. S.H. and M.Z. acknowledge the Karlsruhe School of Optics \& Photonics (KSOP) for support. The blue-light emitting polymer was provided by Merck KGaA. We thank Nanograde Ltd. for supplying $\mathrm{ZnO}$ nanoparticles. The first two authors contributed equally to this work.

\section{References}

1 S. Höfle, A. Schienle, C. Bernhard, M. Bruns, U. Lemmer and A. Colsmann, Adv. Mater., 2014, 26, 5155.

2 Y.-J. Pu, T. Chiba, K. Ideta, S. Takahashi, N. Aizawa, T. Hikichi and J. Kido, Adv. Mater., 2015, 27, 1327.

3 S. Höfle, C. Bernhard, M. Bruns, C. Kübel, T. Scherer, U. Lemmer and A. Colsmann, ACS Appl. Mater. Interfaces, 2015, 7, 8132.

4 T. Chiba, Y.-J. Pu and J. Kido, Adv. Mater., 2015, 27, 4681.

5 F. Nickel, T. Haas, E. Wegner, D. Bahro, S. Salehin, O. Kraft, P. A. Gruber and A. Colsmann, Sol. Energy Mater. Sol. Cells, 2014, 130, 317.

6 D. Angmo, M. Hösel and F. C. Krebs, Sol. Energy Mater. Sol. Cells, 2012, 107, 329.

7 J. E. Carlé, T. R. Andersen, M. Helgesen, E. Bundgaard, M. Jørgensen and F. C. Krebs, Sol. Energy Mater. Sol. Cells, 2013, 108, 126.

8 D. Zhang, K. Ryu, X. Liu, E. Polikarpov, J. Ly, M. E. Tompson and C. Zhou, Nano Lett., 2006, 6, 1880.

9 C. M. Aguirre, S. Auvray, S. Pigeon, R. Izquierdo, P. Desjardins and R. Martel, Appl. Phys. Lett., 2006, 88, 183104.

10 B. F. Zhang, M. Johansson, M. R. Andersson, J. C. Hummelen and O. Inganäs, Adv. Mater., 2002, 14, 662.

11 M.-G. Kang and L. J. Guo, Adv. Mater., 2007, 19, 1391.

12 S.-I. Na, S.-S. Kim, J. Jo and D.-Y. Kim, Adv. Mater., 2008, 20, 4061.

13 M. Cai, Z. Ye, T. Xiao, R. Liu, Y. Chen, R. W. Mayer, R. Biswas, K.-M. Ho, R. Shinar and J. Shinar, Adv. Mater., 2012, 24, 4337. 
14 Y. Zhou, H. Cheun, S. Choi, W. J. Potscavage, C. FuentesHernandez and B. Kippelen, Appl. Phys. Lett., 2010, 97, 153304.

15 A. Colsmann, M. Reinhard, T. H. Kwon, C. Kayser, F. Nickel, J. Czolk, U. Lemmer, N. Clark, J. Jasieniak, A. B. Holmes and D. Jones, Sol. Energy Mater. Sol. Cells, 2012, 98, 118.

16 H. Do, M. Reinhard, H. Vogeler, A. Puetz, M. F. G. Klein, W. Schabel, A. Colsmann and U. Lemmer, Thin Solid Films, 2009, 517, 5900.

17 J. Ouyang, C.-W. Chu, F.-C. Chen, Q. Xu and Y. Yang, Adv. Funct. Mater., 2005, 15, 203.

18 Y. H. Kim, C. Sachse, M. L. Machala, C. May, L. MüllerMeskamp and K. Leo, Adv. Funct. Mater., 2011, 21, 1076.

19 J. Czolk, A. Puetz, D. Kutsarov, M. Reinhard, U. Lemmer and A. Colsmann, Adv. Energy Mater., 2013, 3, 386.

20 M. Reinhard, R. Eckstein, A. Slobodskyy, U. Lemmer and A. Colsmann, Org. Electron., 2013, 14, 273.
21 S. Höfle, A. Schienle, M. Bruns, U. Lemmer and A. Colsmann, Adv. Mater., 2014, 26, 2750.

22 J. Huang, G. Li and Y. Yang, Adv. Mater., 2008, 20, 415.

23 Y.-H. Kim, T.-H. Han, H. Cho, S.-Y. Min, C.-L. Lee and T.-W. Lee, Adv. Funct. Mater., 2014, 24, 3808.

24 Y. Zhou, C. Fuentes-Hernandez, J. Shim, J. Meyer, A. J. Giordano, H. Li, P. Winget, T. Papadopoulos, H. Cheun, J. Kim, M. Fenoll, A. Dindar, W. Haske, E. Najafabadi, T. M. Khan, H. Sojoudi, S. Barlow, S. Graham, J.-L. Brédas, S. R. Marder, A. Kahn and B. Kippelen, Science, 2012, 336, 327.

25 S. Höfle, M. Bruns, S. Strässle, C. Feldmann, U. Lemmer and A. Colsmann, Adv. Mater., 2013, 25, 4113.

26 S. Höfle, T. Lutz, A. Egel, F. Nickel, S. W. Kettlitz, G. Gomard, U. Lemmer and A. Colsmann, ACS Photonics, 2014, 1, 968.

27 S. Höfle, H. Do, E. Mankel, M. Pfaff, Z. Zhang, D. Bahro, T. Mayer, W. Jägermann, D. Gerthsen, C. Feldmann, U. Lemmer and A. Colsmann, Org. Electron., 2013, 14, 1820. 